\title{
PNe abundances: Galactic bulge versus the disc
}

K. Exter (kme@star.ucl.ac.uk)

N. Copernicus Astronomical Centre

M. J. Barlow (mjb@star.ucl.ac.uk)

University College London

N. A. Walton

$I A C$

R. E. S. Clegg

PPARC

Abstract. We compare the abundances (relative to H) of He, N, O, Ar, S, and Ne, determined in a similar way, for a sample of Galactic bulge and a sample of Galactic disc planetary nebulae (PNe). We find that in all aspects - average abundances and dispersion, distribution functions, abundance relationships, and the ratio of $\mathrm{N} / \mathrm{O}-$ the bulge and disc PNe are indistinguishable. Given that it is most likely that the stellar makeup and evolutionary history of the bulge and disc are different, this similarity requires an explanation.

Planetary nebulae abundances give information on both the preceeding stellar evolution (eg. from $\mathrm{N}$ and $\mathrm{He}$ ) and the chemical state of the interstallar medium from which the progenitor star was formed (eg. from $\mathrm{O}, \mathrm{Ne}, \mathrm{S}$, and $\mathrm{Ar}$ ). Comparing the abundances determined for samples of PNe from different parts of the Galaxy can therefore yield much interesting information on the relative evolutionary histories, although due care must be paid to the comparability of the different studies.

For the bulge $\mathrm{PNe}$, an eventual sample of $\sim 45 \mathrm{PNe}$ were analysed for their abundances. The electron temperature $\left(\mathrm{T}_{e}\right)$ and density $\left(\mathrm{n}_{e}\right)$ were determined with the usual method (ratio of [ $\mathrm{O}$ iii] or $[\mathrm{N}$ ii] lines for $\mathrm{T}_{e}$ and $\left[\mathrm{O}\right.$ ii] or $\left[\mathrm{S}\right.$ ii] lines for $\mathrm{n}_{e}$ ) and elemental abundances determine from the ionic abundances via the ICF (ionization correction factor) scheme of Kingsburgh \& Barlow (1994). The abundances for the sample of disc PNe were taken from Kingsburgh \& Barlow (1994), for which the analysis method is the same.

Full details will be published later (Exter et al.) Briefly we find that the average abundances for all elements studied are the same for the bulge and disc samples, as within the errors are also the dispersions. The distribution functions of $\mathrm{O}, \mathrm{N}, \mathrm{N} / \mathrm{O}$, and He are also the same, and all relationships between these elements are statisitically indistinguishable for the two samples. We particularly emphasize the similiarity in these aspects for the ratio of $\mathrm{N} / \mathrm{O}$, which is usually taken as an indicator of the precursor mass, and in the oxygen abundance for the

(C) 2020 Kluwer Academic Publishers. Printed in the Netherlands.

Exter01_bulgePNe.tex; 23/11/2020; 15:32; p.1 
Type I (from higher mass progenitor stars) and non-Type I PNe; no depletion of oxygen (or any other element) for the Type I PNe is found. We find a 15-20\% ratio of occurance of Type I PNe in our two samples, within the errors the same. Finally, we find that with respect to the stellar distributions (in $[\mathrm{Fe} / \mathrm{H}]$ converted to $[\mathrm{O} / \mathrm{H}]$ for $\mathrm{K}$ III and $\mathrm{G} \mathrm{V}$ stars), the PNe are deficient at the high metallicity end and the peak of the distributions are shifted lower by 0.1-0.2 dex.

Taken at face value our results imply that the bulge and disc PNe come from the same precursor populations with the same initial chemistry and mass distribution. This is at odds with the generally understood view that the bulge consists of lower mass stars and begun star formation before did the disc. Our results are not incompatable with this view, but more detailed analysis is deferred to Exter et al.

The scatter in the nitrogen abundances is wider than that caused by errors, reflecting the mass range of the precursor stars. No other element shows this effect. The Type I PNe, chosen on the basis only of the $\mathrm{N} / \mathrm{O}$ ratio, show $\mathrm{He} / \mathrm{H}$ values that cover the same range as do the non-Type I PNe, implying that if the $\mathrm{N} / \mathrm{O}$ criterium is appropriate (and is simply linked to progenitor mass), the helium enrichement is not such a good indicator of the progenitor mass.

\section{References}

Kingsburgh, R.L., Barlow, M.J: 1994, Mon. Not. R. Astron. Soc. 271,257

Address for Offprints: M.J. Barlow

Physics and Astronomy, University College London

Gower Str., London WC1E 6BT

UK 